\title{
CONSIDERING VARIANCES OF QUASI-RANDOM EFFECTS IN RELATIVE GPS POSITIONING PERFORMED DURING DAYTIME AND NIGHTTIME PERIODS - A NOVEL TWO-STAGE APPROACH
}

\author{
Darko ANĐIĆ* \\ Department of State Survey and Mapping, Real Estate Administration, Podgorica, Montenegro
}

Received 21 March 2020; accepted 12 March 2021

\begin{abstract}
In this paper, a new two-stage approach, involving an integral treatement of all quasi-random effects limiting the accuracy of relative GPS positioning and the method of moments to obtain final variance components regarding the effects of short-term ("far-field") multipath (factor $\beta$ ), joint action of long-term ("near-field") multipath and receiver antenna phase center offset and variations (factor $\alpha 1$ ), as well as joint action of tropospheric and ionospheric refraction (factor $\alpha 2$ ), is presented. In the study, GPS data collected on five baselines were used. Variance components of the quasirandom effects were obtained for the three relative GPS coordinates $(e, n$ and $u$ ) using individually monthly datsets including daytime- and those including nighttime-wise ambiguity-fixed baseline solutions. The related results show that statistically significant inequality exists when comparing corresponding variances obtained for daytime and nighttime periods. It turned out that the following standard deviation estimates intervals are present (by the coordinates $e, n$ and $u$, respectively): (a) daytime period: 3.3-6.9, 4.6-9.0 and 9.1-20.3 mm (factor $\beta$ ); 1.5-4.7, 1.9-7.0 and 3.4-21.9 mm (factor $\alpha 1$ ); 0.0116$0.3282,0.0103-0.2365$ and $0.1222-0.7818 \mathrm{~mm} / \mathrm{km}$ (factor $\alpha 2$ ); (b) nighttime period: $3.2-4.9,4.7-7.3$ and $8.4-15.4 \mathrm{~mm}$ (factor $\beta$ ); $0.8-3.8,2.1-5.0$ and $3.1-15.8 \mathrm{~mm}$ (factor $\alpha 1$ ); 0.0118-0.2734, 0.0097-0.2289 and $0.0752-0.6315 \mathrm{~mm} / \mathrm{km}($ factor $\alpha 2)$.
\end{abstract}

Keywords: relative GPS positioning, quasi-random effects, variance components, ANOVA estimates, method of moments, sub-daily impacts, statistical hypotheses testing.

\section{Introduction}

Many studies have been conducted with an aim to consider and represent statistical behaviour of unmodelled, i.e. residual effects arising in the relative GPS positioning. But, in each of them these effects were treated individually and a reader can be referred to the recent ones, such as, e.g. Hu et al. (2015), Deng et al. (2016), Elsobeiey and El-Diasty (2016), Jadviščok et al. (2016), Klos et al. (2018), Zhou et al. (2018), Anđić, (2019b), Han et al. (2019), Juni and Rózsa (2019), Kallio et al. (2019).

Herein, however, the author uses a linear model involving effects limiting relative GPS positioning accuarcy at once. Namely, the effects, such as short- "ffar-field" multipath - FF MP) and long-term ("near-field" multipath - NF MP, receiver antenna phase center offset and variations - RAPCOV, tropospheric and ionospheric refraction - TI) unmodelled effects, are the only ones that must not be neglected when making a choice of baselines as in this study (each of them is of less than $300 \mathrm{~km}$ in length). So, in this regard, only the previously mentioned effects are being considered in this study.

In the previous work, related to the use of the twoway nested classification in GPS positioning, published by Anđić (2016, 2019a), only a baseline of $40 \mathrm{~km}$ in length was considered, and only ANOVA estimates obtained on the basis of daily datasets were presented. Herein, a further analysis, dealing with ANOVA estimates obtained by using sub-daily (daytime and nighttime) datasets is performed. In addition, one more method (method of moments, as a tool which the second stage of the approach applied here is based on), as well as GPS data collected at ten ending stations two of which belong to MontePos (Montenegrin Permanent Network) (PODG and BAR), and remaining eight that have been integrated in EPN (EUREF Permanent Network) infrastructure (TORI, IENG, IGMI, PRAT, BOR1, WROC, BACA and BAIA). These stations establish five baselines, ranging from 5.6 to $281.9 \mathrm{~km}$ in length. All of the ten stations have been equipped with high-quality antennas, mounted in different way and surrounded with

*Corresponding author. E-mail: andjic.darko@gmail.com 
potential reflectors of different characteristics, some of which were in the near vicinity while others were far away from an antenna. More detailed information related to the two MontePos stations can be found at the following link: http://www.nekretnine.co.me/me/Djelatnosti5.asp. On the other side, information regarding eight EPN stations are provided at the following address: http://epncb.oma. be/_networkdata/stationmaps.php.

It should be noted that no paper dealing with the previously mentioned effects in the way presented in this study has been published so far. That is where the motivation for writing this paper came from.

\section{Data and methods}

\subsection{Input data}

For the purpose of the study, a total number of 38548930 true errors (corresponding to the relative coordinates $e, n$ and $u$ ) remained after outliers removal, were used. These solutions were obtained by using Trimble Total Control software in processing $0.033-\mathrm{Hz}$ GPS data, collected, as previously said, at ending stations of five baselines. The baselines are located in Italy (TORI-IENG and IGMIPRAT, being 5.6 and $13.6 \mathrm{~km}$ long, respectively), in Montenegro (BAR-PODG, $40 \mathrm{~km}$ long), in Poland (BOR1WROC, $129.5 \mathrm{~km}$ long) and in Romania (BACA-BAIA, of $281.9 \mathrm{~km}$ in length).

In data processing, the processing interval of $30 \mathrm{~s}$, precise orbits, elevation cutoff of $10^{\circ}$, ionosphere-free linear combination, default calibration model, Saastamoinen tropospheric model, MSIS meteorological model and OTF processing mode (as an auxiliary one) were used. Such settings allowed all unmodelled effects to be expressed in each ambiguity-fixed solution.

\subsection{The two-stage method used in the study}

In the first stage of the study, the two-way nested ANOVA was applied individually on monthly datasets of daytimeand nighttime-wise true errors for all of the three relative coordinates ( $e, n$ and $u$ ), whereby variance components estimates $m_{\varepsilon, c}^{2}=\widehat{\sigma_{\varepsilon, c}^{2}}, m_{\beta, c}^{2}=\widehat{\sigma_{\beta, c}^{2}}$ and $m_{\alpha, c}^{2}=\widehat{\sigma_{\alpha, c}^{2}}$, with $c \in\{e, n, u\}$, were calculated. Herein, the following denotations were introduced: $\alpha$ - the nesting factor (related to the unmodelled joint long-term effect, including NF MP, RAPCOV, TI); $\beta$ - the nested factor, nested within $\alpha$ (related to the unmodelled short-term effect, i.e. FF MP); $\varepsilon$ - the purely random error, nested within $\beta$. It should also be mentioned that the 3-minute constancy of the short-term effect (i.e. FF MP - the nested factor $\beta$ ) and the 90-minute constancy of all long-term effects (i.e. NF MP, RAPCOV, TI - the nesting factor $\alpha$ ) were assumed in the calculations.

The concept of the first-stage-related method is shown in Figure 1.

Namely, here we introduce the true error model equation of an individual coordinate $c$ as follows:

$$
\Delta_{c, i j k}=\alpha_{c, i}+\beta_{c, j(i)}+\varepsilon_{c, k(i j)},
$$

whereby the accompanying stochastic model is based on the assumptions below:

$$
\begin{aligned}
& \alpha_{c, i} \sim N\left(0, \sigma_{\alpha, c}^{2}\right), \text { with } i \in\{1,2, \ldots, a\}, a \geq 2, \\
& \beta_{c, j(i)} \sim N\left(0, \sigma_{\beta, c}^{2}\right), \text { with } j \in\left\{1,2, \ldots, b_{i}\right\}, b_{i} \geq 2, \\
& \varepsilon_{c, k(i j)} \sim N\left(0, \sigma_{\varepsilon, c}^{2}\right), \text { with } k \in\left\{1,2, \ldots, n_{i j}\right\}, n_{i j} \geq 2,(2 \\
& \operatorname{Cov}\left(\varepsilon_{c, k(i j)}, \beta_{c, j(i)}\right)=\operatorname{Cov}\left(\varepsilon_{c, k(i j)}, \alpha_{c, i}\right)= \\
& \operatorname{Cov}\left(\beta_{c, j(i)}, \alpha_{c, i}\right)=0, \\
& \operatorname{Cov}\left(\varepsilon_{c, k(i j)}, \varepsilon_{c, r(p q)}\right)=0, \text { where } i \neq p \vee j \neq q \vee k \neq r .
\end{aligned}
$$

On the basis of (1) and (2a-e), one writes:

$$
\operatorname{Var}\left(\Delta_{c, i j k}\right) \equiv \sigma_{\Delta}^{2}=\sigma_{\alpha, c}^{2}+\sigma_{\beta, c}^{2}+\sigma_{\varepsilon, c}^{2} \text {, }
$$

whereby $\sigma_{\alpha, c}^{2}, \sigma_{\beta, c}^{2}$ and $\sigma_{\varepsilon, c}^{2}$ represent the variance components that are to be estimated in the ANOVA estimation procedure. With of an aim to avoid unnecessary transcription of the corresponding formulas, that procedure is not shown herein, so, for details, a reader is reffered to Anđić (2016).

In the second stage, with the aim of calculation of final variance components, an iterative method of moments was used. Namelly, as assumptions regarding unmodelled and purely random effects are $\left(i \in\{1,2, \ldots, a\}, j \in\left\{1,2, \ldots, b_{i}\right\}\right.$, $\left.k \in\left\{1,2, \ldots, n_{i j}\right\}\right)$ :

$$
\left\{\begin{array}{lll}
\alpha_{c, i} & \beta_{c, j(i)} & \varepsilon_{c, k(i j)}
\end{array}\right\}^{\mathrm{T}} \sim N\left(\mathbf{0}_{3 \times 1}, \operatorname{diag}\left\{\sigma_{\alpha, c}^{2} \quad \sigma_{\beta, c}^{2} \quad \sigma_{\varepsilon, c}^{2}\right\}\right),(4)
$$

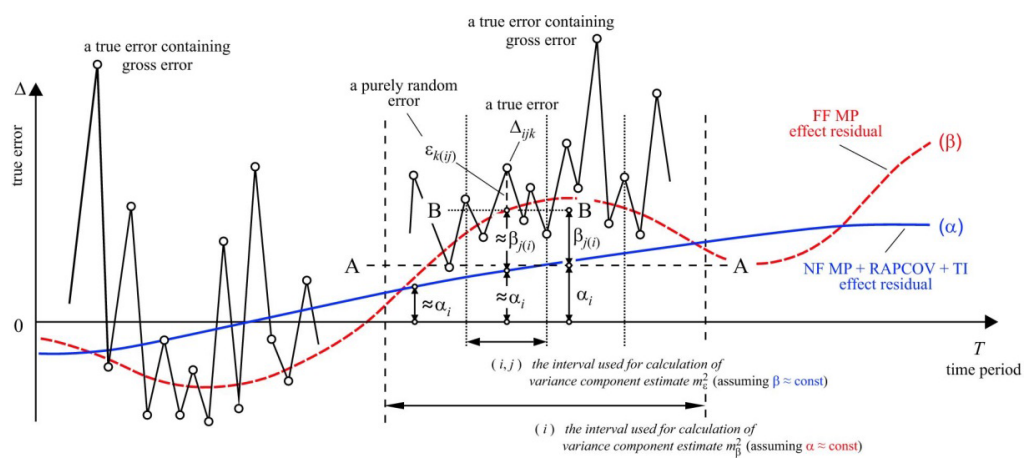

Figure 1. The concept of the method applied herein (adapted from Figure 6.3.1 in Anđić, 2019b) 
then one can establish the second and the fourth $\xi_{c, s}$ moment for those effects as follows $(\xi \in\{\alpha, \beta, \varepsilon\}, s \in\{1,2, \ldots, g\}$, $g \geq k)$ :

$$
\begin{aligned}
& \mathrm{M}\left\{\xi_{c, s}^{2}\right\}=\sigma_{\xi, c}^{2}=\sigma_{1 ; \xi, c}^{2} Q_{1, c, s s}+\ldots+\sigma_{k ; \xi, c}^{2} Q_{k, c, s s} ; \\
& \mathrm{D}\left\{\xi_{c, s}^{2}\right\}=\sigma_{\xi_{c, s}^{2}}^{2}=2 \sigma_{\xi, c ; s}^{4},
\end{aligned}
$$

where $\sigma_{1 ; \xi, c}^{2}, \ldots, \sigma_{k ; \xi, c}^{2}$ are the unknown variance components that are estimated.

For that purpose in this study, considering, in addition, that $\mathrm{M}\left\{\xi_{c, s}^{2}\right\}=\mathrm{M}\left\{m_{\xi, c ; s}^{2}\right\}$, one establishes the linear and stochastic model of the standard least squares as follows:

$$
\begin{aligned}
& v_{\alpha, c ; s}=\sigma_{1 ; \alpha, c}^{2}+\sigma_{2 ; \alpha, c}^{2} D_{s[k m]}^{2}-m_{\alpha, c ; s}^{2} ; \\
& v_{\beta, c ; s}=\sigma_{1 ; \beta, c}^{2}-m_{\beta, c ; s}^{2} ; \\
& v_{\varepsilon, c ; s}=\sigma_{1 ; \varepsilon, c}^{2}-m_{\varepsilon, c ; s}^{2} ; \\
& M\left\{v_{\alpha, c ; s}\right\}=0, \quad P_{\alpha, c ; s}=N_{\alpha, c ; s} /\left(2 \sigma_{\alpha, c ; s}^{4}\right) ; \\
& M\left\{v_{\beta, c ; s}\right\}=0, \quad P_{\beta, c ; s}=N_{\beta, c ; s} /\left(2 \sigma_{\beta, c ; s}^{4}\right) ; \\
& M\left\{v_{\varepsilon, c ; s}\right\}=0, \quad P_{\varepsilon, c ; s}=N_{\varepsilon, c ; s} /\left(2 \sigma_{\varepsilon, c ; s}^{4}\right),
\end{aligned}
$$

where $v_{\xi, c ; s}, P_{\xi, c ; s}, N_{\xi, c ; s}$ and $D_{s}(\xi \in\{\alpha, \beta, \varepsilon\}, c \in\{e, n, u\}$, $s \in\{1,2,3,4,5\})$ are denotations for corrections, weights, numbers of daytime- or nighttime-wise true errors within a monthly dataset based on which ANOVA estimates $m_{\xi, c ; s}^{2}$ were obtained, and baseline lengths, respectively.

The unknown variance components $\sigma_{1 ; \alpha, c}^{2}, \sigma_{2 ; \alpha, c}^{2}$, $\sigma_{1 ; \beta, c}^{2}$ and $\sigma_{1 ; \varepsilon, c}^{2}$, regarding, respectively, the joint effect of NF MP and RAPCOV, the joint effect TI, the effect of FF MP and the purely random error, are estimated through the following iterative procedure:

Step 1. Adopting initial weight matrices:

$$
\mathbf{P}_{\alpha, c}^{(0)}=\mathbf{P}_{\beta, c}^{(0)}=\mathbf{P}_{\varepsilon, c}^{(0)} \equiv \mathbf{I}_{5 \times 5} .
$$

Step 2. Calculating initial estimates of variance components:

$$
\begin{aligned}
& {\left[\begin{array}{l}
\widehat{\sigma_{1 ; \alpha, c}^{2(0)}} \\
\overline{\sigma_{2 ; \alpha, c}^{2(0)}}
\end{array}\right] \equiv\left[\begin{array}{l}
m_{1 ; \alpha, c}^{2(0)} \\
m_{2 ; \alpha, c}^{2(0)}
\end{array}\right]=\left(\mathbf{A}^{\mathrm{T}} \mathbf{P}_{\alpha, c}^{(0)} \mathbf{A}\right)^{-1} \mathbf{A}^{\mathrm{T}} \mathbf{P}_{\alpha, c}^{(0)} \mathbf{1}_{\alpha, c} ;} \\
& \widehat{\sigma_{1 ; \beta, c}^{2(0)}} \equiv m_{1 ; \beta, c}^{2(0)}=\left(\mathbf{1}_{5 \times 1}^{\mathrm{T}} \mathbf{P}_{\beta, c}^{(0)} \mathbf{1}_{5 \times 1}\right)^{-1} \mathbf{1}_{5 \times 1}^{\mathrm{T}} \mathbf{P}_{\beta, c}^{(0)} \mathbf{1}_{\beta, c} \\
& \widehat{\sigma_{1 ; \varepsilon, c}^{2(0)}} \equiv m_{1 ; \varepsilon, c}^{2(0)}=\left(\mathbf{1}_{5 \times 1}^{\mathrm{T}} \mathbf{P}_{\varepsilon, c}^{(0)} \mathbf{1}_{5 \times 1}\right)^{-1} \mathbf{1}_{5 \times 1}^{\mathrm{T}} \mathbf{P}_{\varepsilon, c}^{(0)} \mathbf{1}_{\varepsilon, c},
\end{aligned}
$$

whereby, besides the vector of ones $\left(\mathbf{1}_{5 \times 1}\right)$, we have the following matrix and vectors $(\xi \in\{\alpha, \beta, \varepsilon\})$ :

$$
\begin{aligned}
& \mathbf{A}=\left[\begin{array}{ll}
\mathbf{1}_{5 \times 1} & \mathbf{d}
\end{array}\right] ;
\end{aligned}
$$

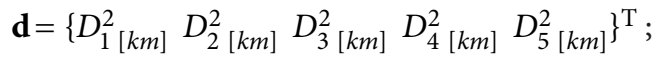

$$
\mathbf{l}_{\xi, c}=\left\{m_{\xi, c ; 1}^{2} m_{\xi, c ; 2}^{2} m_{\xi, c ; 3}^{2} m_{\xi, c ; 4}^{2} m_{\xi, c ; 5}^{2}\right\}^{\mathrm{T}}
$$

Step 3. Calculating weight matrices using variance components estimates obtained in Step 2:

$$
\begin{aligned}
& \mathbf{P}_{\alpha, c}^{(1)}=\operatorname{diag}\left\{N_{\alpha, c ; s} /\left(m_{1 ; \alpha, c}^{2(0)}+m_{2 ; \alpha, c}^{2(0)} D_{s[k m]}^{2}\right)^{2}\right\} ; \\
& \mathbf{P}_{\beta, c}^{(1)}=\operatorname{diag}\left\{N_{\beta, c ; s} / m_{1 ; \beta, c}^{4(0)}\right\} ; \\
& \mathbf{P}_{\varepsilon, c}^{(1)}=\operatorname{diag}\left\{N_{\varepsilon, c ; s} / m_{1 ; \varepsilon, c}^{4(0)}\right\} .
\end{aligned}
$$

Step 4. Calculating new (final) estimates of variance components:

$$
\begin{aligned}
& {\left[\begin{array}{l}
\widehat{\sigma_{1 ; \alpha, c}^{2(1)}} \\
\overline{\sigma_{2 ; \alpha, c}^{2(1)}}
\end{array}\right] \equiv\left[\begin{array}{c}
m_{1 ; \alpha, c}^{2(1)} \\
m_{2 ; \alpha, c}^{2(1)}
\end{array}\right]=\left(\mathbf{A}^{\mathrm{T}} \mathbf{P}_{\alpha, c}^{(1)} \mathbf{A}\right)^{-1} \mathbf{A}^{\mathrm{T}} \mathbf{P}_{\alpha, c}^{(1)} \mathbf{1}_{\alpha, c} ;} \\
& \widehat{\sigma_{1 ; \beta, c}^{2(1)}} \equiv m_{1 ; \beta, c}^{2(1)}=\left(\mathbf{1}_{5 \times 1}^{\mathrm{T}} \mathbf{P}_{\beta, c}^{(1)} \mathbf{1}_{5 \times 1}\right)^{-1} \mathbf{1}_{5 \times 1}^{\mathrm{T}} \mathbf{P}_{\beta, c}^{(1)} \mathbf{1}_{\beta, c} ; \\
& \widehat{\sigma_{1 ; \varepsilon, c}^{2(1)}} \equiv m_{1 ; \varepsilon, c}^{2(1)}=\left(\mathbf{1}_{5 \times 1}^{\mathrm{T}} \mathbf{P}_{\varepsilon, c}^{(1)} \mathbf{1}_{5 \times 1}\right)^{-1} \mathbf{1}_{5 \times 1}^{\mathrm{T}} \mathbf{P}_{\varepsilon, c}^{(1)} \mathbf{1}_{\varepsilon, c} .
\end{aligned}
$$

Step 5. Repeating steps 3 and 4 until the following conditions are met ( $\tau$ denotes an iteration number):

$$
\begin{aligned}
& \left|m_{1 ; \alpha(\beta)(\varepsilon), c}^{2(\tau+1)}-m_{1 ; \alpha(\beta)(\varepsilon), c}^{2(\tau)}\right| \leq 10^{-1} ; \\
& \left|m_{2 ; \alpha, c}^{2(\tau+1)}-m_{2 ; \alpha, c}^{2(\tau)}\right| \leq 10^{-1} .
\end{aligned}
$$

Step 6. Adopting final estimates $m_{1 ; \alpha, c}^{2}, m_{2 ; \alpha, c}^{2}, m_{1 ; \beta, c}^{2}$ and $m_{1 ; \varepsilon, c}^{2}$, with the corresponding degrees of freedom, calculated as $f_{1,2 ; \alpha, c}=\sum_{s=1}^{5} f_{\alpha, c ; s}$ (for the first two), $f_{1 ; \beta, c}=\sum_{s=1}^{5} f_{\beta, c ; s}$ and $f_{1 ; \varepsilon, c}=\sum_{s=1}^{5} f_{\varepsilon, c ; s}$. Degrees of freedom $f_{\xi, c ; s}, \quad \xi \in\{\alpha, \beta, \varepsilon\}$, are calculated as shown in Anđić $(2016,2019 a)$.

\section{Results}

In Tables 1 to 6 , the results of ANOVA estimation performed for the daytime and nighttime within the fouryear period considered (2008-2011), are given. The results are square roots of epoch-wise variance components estimates (i.e. standard deviations estimates) obtained using individually monthly datasets including daytime- and those including nighttime-wise true errors. In doing so, a linear model based on two-way nested classification with random effects with no interactions was used (see Anđić, 2016, 2019a).

Square roots of the final epoch-wise variance components estimates, by months within the period 2008-2011, are presented graphically in Figures 2 to 5. 
Table 1. Extreme values of epoch-wise standard deviations estimates for the coordinate $e$ and the daytime $(D T)$ period

\begin{tabular}{|l|c|c|c|c|c|c|}
\hline \multirow{2}{*}{ Baseline } & \multicolumn{2}{|c|}{$m_{\varepsilon, e[m m]}^{D T}$} & \multicolumn{2}{c|}{$m_{\beta, e[m m}^{D T}$} & \multicolumn{2}{c|}{$m_{\alpha, e[m m]}^{D T}$} \\
\cline { 2 - 7 } & $\min$ & $\max$ & $\min$ & $\max$ & $\min$ & $\max$ \\
\hline TORI-IENG & 2.1 & 2.9 & 2.5 & 4.9 & 1.4 & 4.8 \\
\hline IGMI-PRAT & 3.2 & 4.1 & 2.5 & 5.0 & 1.8 & 4.7 \\
\hline BAR-PODG & 3.2 & 4.9 & 3.4 & 6.1 & 2.2 & 6.6 \\
\hline BOR1-WROC & 2.6 & 3.3 & 2.7 & 5.7 & 1.9 & 7.4 \\
\hline BACA-BAIA & 2.6 & 3.5 & 4.0 & 15.2 & 4.7 & 235.6 \\
\hline
\end{tabular}

Table 2. Extreme values of epoch-wise standard deviations estimates for the coordinate $n$ and the daytime (DT) period

\begin{tabular}{|l|c|c|c|c|c|c|}
\hline \multirow{2}{*}{ Baseline } & \multicolumn{2}{|c|}{$m_{\varepsilon, n[\mathrm{~mm}]}^{D T}$} & \multicolumn{2}{c|}{$m_{\beta, n[\mathrm{~mm}]}^{D T}$} & \multicolumn{2}{c|}{$m_{\alpha, n[\mathrm{~mm}]}^{D T}$} \\
\cline { 2 - 7 } & $\min$ & $\max$ & $\min$ & $\max$ & $\min$ & $\max$ \\
\hline TORI-IENG & 2.5 & 3.9 & 2.9 & 8.3 & 1.5 & 8.4 \\
\hline IGMI-PRAT & 3.7 & 5.7 & 2.9 & 7.1 & 1.9 & 6.6 \\
\hline BAR-PODG & 4.2 & 6.6 & 4.4 & 7.9 & 2.3 & 9.2 \\
\hline BOR1-WROC & 3.5 & 4.8 & 3.1 & 7.5 & 3.9 & 10.8 \\
\hline BACA-BAIA & 3.6 & 4.8 & 5.6 & 16.8 & 4.3 & 131.4 \\
\hline
\end{tabular}

Table 3. Extreme values of epoch-wise standard deviations estimates for the coordinate $u$ and the daytime (DT) period

\begin{tabular}{|l|c|c|c|c|c|c|}
\hline \multirow{2}{*}{ Baseline } & \multicolumn{2}{|c|}{$m_{\varepsilon, u}^{D T}[\mathrm{~mm}]$} & \multicolumn{2}{c|}{$m_{\beta, u}^{D T}[\mathrm{~mm}]$} & \multicolumn{2}{c|}{$m_{\alpha, u}^{D T}[\mathrm{~mm}]$} \\
\cline { 2 - 7 } & $\min$ & $\max$ & $\min$ & $\max$ & $\min$ & $\max$ \\
\hline TORI-IENG & 5.1 & 8.0 & 6.8 & 16.6 & 3.7 & 16.2 \\
\hline IGMI-PRAT & 8.8 & 11.0 & 7.4 & 14.4 & 6.1 & 23.0 \\
\hline BAR-PODG & 8.2 & 13.5 & 9.1 & 17.0 & 13.5 & 38.7 \\
\hline BOR1-WROC & 6.8 & 8.5 & 7.3 & 15.7 & 16.4 & 53.6 \\
\hline BACA-BAIA & 6.5 & 8.6 & 10.5 & 40.6 & 33.1 & 326.4 \\
\hline
\end{tabular}

Table 4. Extreme values of epoch-wise standard deviations estimates for the coordinate $e$ and the nighttime (NT) period

\begin{tabular}{|l|c|c|c|c|c|c|}
\hline \multirow{2}{*}{ Baseline } & \multicolumn{2}{|c|}{$m_{\varepsilon, e[m]}^{N T}$} & \multicolumn{2}{c|}{$m_{\beta, e[m m]}^{N T}$} & \multicolumn{2}{c|}{$m_{\alpha, e[m m]}^{N T}$} \\
\cline { 2 - 8 } & $\min$ & $\max$ & $\min$ & $\max$ & $\min$ & $\max$ \\
\hline TORI-IENG & 2.1 & 2.7 & 2.3 & 4.8 & 1.4 & 4.6 \\
\hline IGMI-PRAT & 3.2 & 3.9 & 2.2 & 4.4 & 1.1 & 4.1 \\
\hline BAR-PODG & 3.3 & 4.7 & 3.4 & 5.3 & 1.6 & 4.3 \\
\hline BOR1-WROC & 2.6 & 3.1 & 2.7 & 4.4 & 1.8 & 5.9 \\
\hline BACA-BAIA & 2.7 & 3.1 & 3.9 & 7.8 & 4.3 & 157.6 \\
\hline
\end{tabular}

Table 5. Extreme values of epoch-wise standard deviations estimates for the coordinate $n$ and the nighttime (NT) period

\begin{tabular}{|l|c|c|c|c|c|c|}
\hline \multirow{2}{*}{ Baseline } & \multicolumn{2}{|c|}{$m_{\varepsilon, n[m]}^{N T}$} & \multicolumn{2}{c|}{$m_{\beta, n}^{N T}[\mathrm{~mm}]$} & \multicolumn{2}{c|}{$m_{\alpha, n}^{N T}[\mathrm{~mm}]$} \\
\cline { 2 - 8 } & $\min$ & $\max$ & $\min$ & $\max$ & $\min$ & $\max$ \\
\hline TORI-IENG & 2.4 & 3.3 & 3.1 & 8.0 & 1.7 & 6.9 \\
\hline IGMI-PRAT & 4.0 & 5.6 & 3.1 & 5.8 & 2.1 & 5.1 \\
\hline BAR-PODG & 4.4 & 6.1 & 4.5 & 6.5 & 2.2 & 5.5 \\
\hline BOR1-WROC & 3.6 & 4.4 & 3.8 & 5.6 & 3.7 & 14.0 \\
\hline BACA-BAIA & 3.7 & 4.3 & 6.3 & 12.9 & 5.0 & 125.2 \\
\hline
\end{tabular}

Table 6. Extreme values of epoch-wise standard deviations estimates for the coordinate $u$ and the nighttime (NT) period

\begin{tabular}{|l|c|c|c|c|c|c|}
\hline \multirow{2}{*}{ Baseline } & \multicolumn{2}{|c|}{$m_{\varepsilon, u}^{N T}[\mathrm{~mm}]$} & \multicolumn{2}{c|}{$m_{\beta, u}^{N T}[\mathrm{~mm}]$} & \multicolumn{2}{c|}{$m_{\alpha, u}^{N T}[\mathrm{~mm}]$} \\
\cline { 2 - 7 } & $\min$ & $\max$ & $\min$ & $\max$ & $\min$ & $\max$ \\
\hline TORI-IENG & 5.3 & 6.4 & 6.4 & 15.7 & 2.9 & 16.3 \\
\hline IGMI-PRAT & 8.7 & 11.3 & 7.3 & 12.8 & 7.2 & 16.7 \\
\hline BAR-PODG & 8.0 & 13.1 & 9.0 & 14.8 & 12.7 & 35.3 \\
\hline BOR1-WROC & 6.5 & 8.0 & 6.7 & 12.8 & 15.1 & 62.4 \\
\hline BACA-BAIA & 6.5 & 8.0 & 10.0 & 27.0 & 24.0 & 237.9 \\
\hline
\end{tabular}
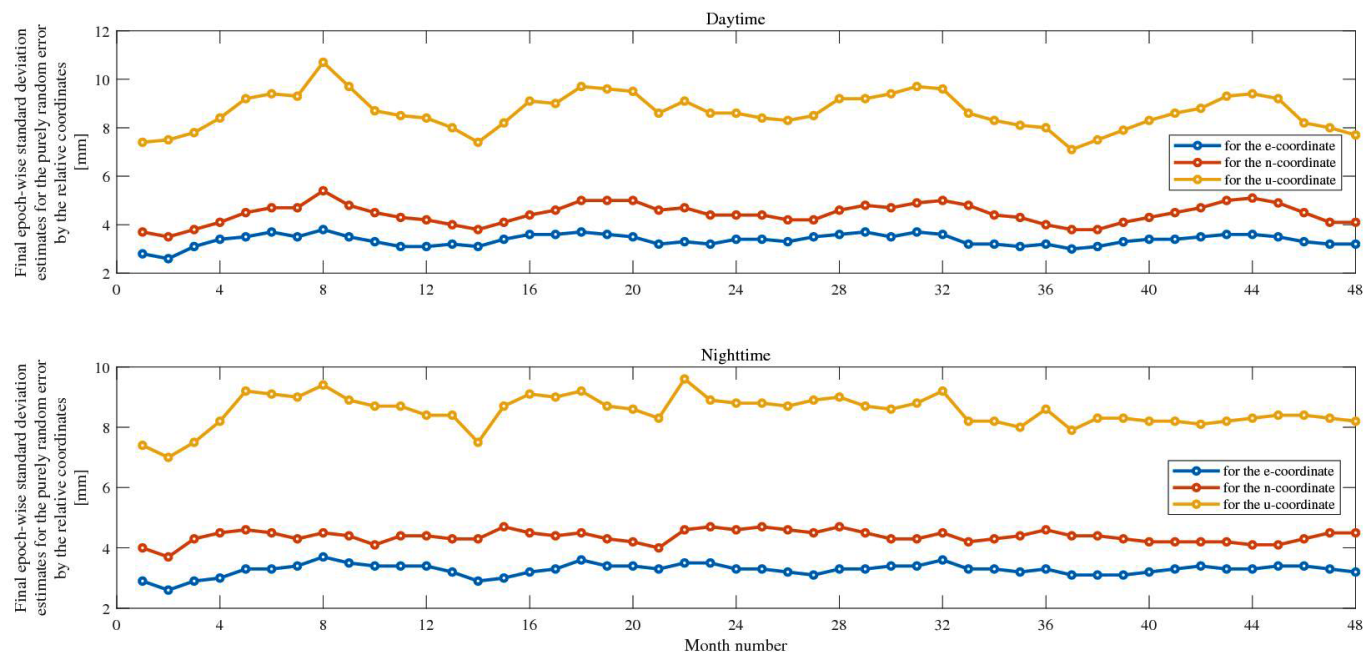

Figure 2. Final epoch-wise standard deviation estimates by the relative coordinates $(e, n$ and $u)$ for the purely random error, obtained for the daytime and the nighttime period 

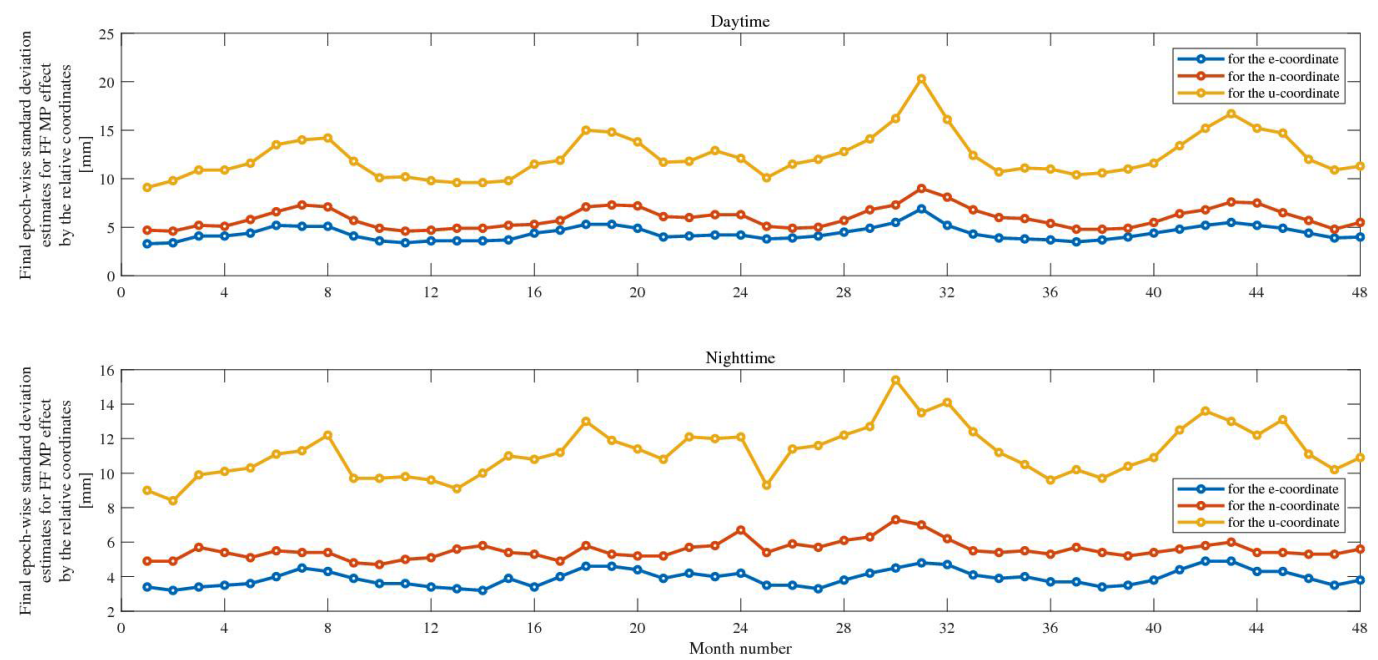

Figure 3. Final epoch-wise standard deviation estimates by the relative coordinates $(e, n$ and $u)$ for the effect of FF MP, obtained for the daytime and the nighttime period
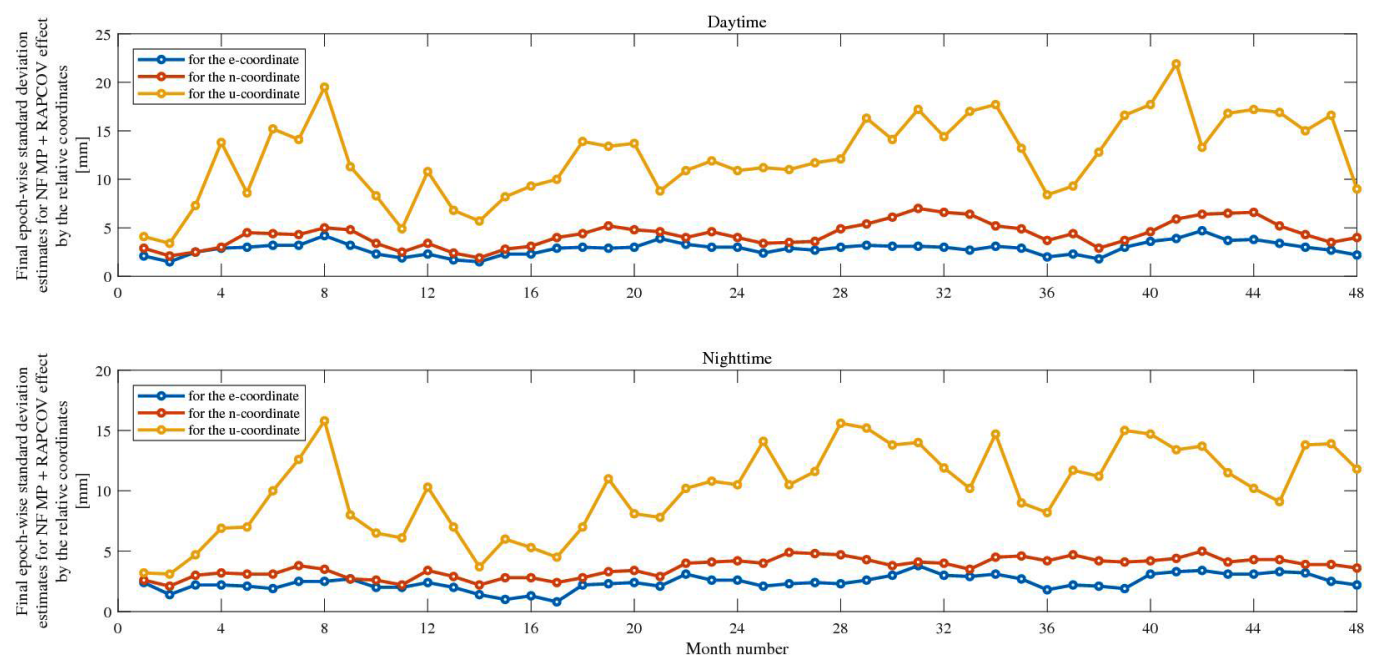

Figure 4. Final epoch-wise standard deviation estimates by the relative coordinates $(e, n$ and $u)$ for the joint effect of NF MP and RAPCOV, obtained for the daytime and the nighttime period
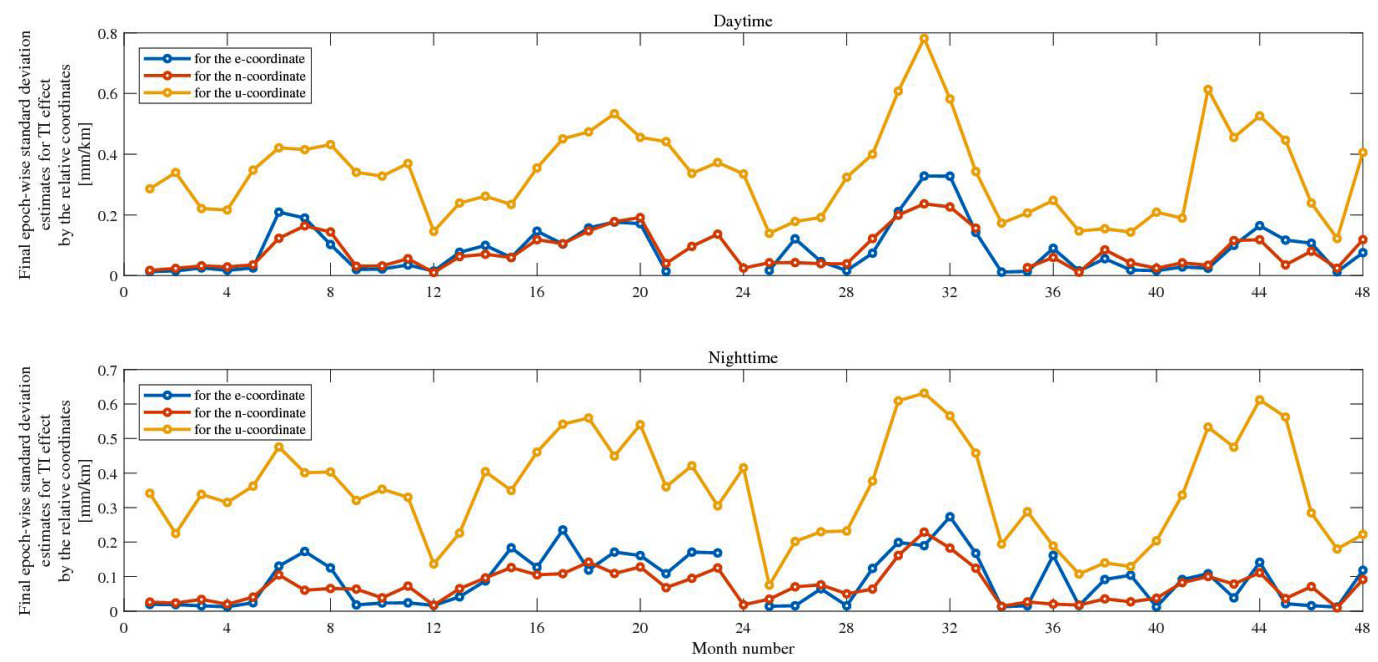

Figure 5. Final epoch-wise standard deviation estimates by the relative coordinates (e, $n$ and $u)$ for the joint effect of TI, obtained for the daytime and the nighttime period 
After applying the well known F-test for equality of two variances (for details, a reader can see, e.g. Snedecor \& Cochran, 1989), herein based on the testing:

(a) $H_{0 ; 1}: \sigma_{1 ; \alpha(\beta), c ; D T}^{2}=\sigma_{1 ; \alpha(\beta), c ; N T}^{2}$,

against $H_{a 1 ; 1}: \sigma_{1 ; \alpha(\beta), c ; D T}^{2}>\sigma_{1 ; \alpha(\beta), c ; N T}^{2}$,

for $m_{1 ; \alpha(\beta), c ; D T}^{2}>m_{1 ; \alpha(\beta), c ; N T}^{2}$,

as well as:

$$
\begin{aligned}
& H_{a 2 ; 1}: \sigma_{1 ; \alpha(\beta), c ; D T}^{2}<\sigma_{1 ; \alpha(\beta), c ; N T}^{2}, \\
& \text { for } m_{1 ; \alpha(\beta), c ; D T}^{2}<m_{1 ; \alpha(\beta), c ; N T}^{2},
\end{aligned}
$$

and also:

$$
\text { (b) } H_{0 ; 2}: \sigma_{2 ; \alpha, c ; D T}^{2}=\sigma_{2 ; \alpha, c ; N T}^{2} \text {, against }
$$

$$
H_{a 1 ; 2}: \sigma_{2 ; \alpha, c ; D T}^{2}>\sigma_{2 ; \alpha, c ; N T}^{2} \text {, for } m_{2 ; \alpha, c ; D T}^{2}>m_{2 ; \alpha, c ; N T}^{2},
$$

as well as:

$$
H_{a 2 ; 2}: \sigma_{2 ; \alpha, c ; D T}^{2}<\sigma_{2 ; \alpha, c ; N T}^{2} \text {, for } m_{2 ; \alpha, c ; D T}^{2}<m_{2 ; \alpha, c ; N T}^{2},
$$

(DT and NT were introduced to denote daytime and nighttime).

So, adopting the $5 \%$ significance level, one obtained the results that are given in Table 7 .

\section{Conclusions}

This study, based on a very large and representative sample, showed that there is statistically significant inequality when comparing corresponding variances of unmodelled effects (limiting relative GPS positioning accuracy) obtained for daytime and nighttime periods (see Table 7).

When it comes to extreme values of the final epochwise standard deviation estimates for the effects considered, one can be spotted that a seasonal pattern is also present, especially pronounced with the joint effect of TI (see Figures 2 to 5). These extreme values (with the related degrees of freedom), as well as the corresponding mean values of those standard deviations estimates are given in the continuation by the relative coordinates ( $e, n$ and $u)$.

"Far-field" multipath effect:

Daytime period

- $\min m_{1 ; \beta, e}^{D T}=3.3 \mathrm{~mm}(19198.65$ d.f $)$;

$$
\begin{aligned}
& \max m_{1 ; \beta, e}^{D T}=6.9 \mathrm{~mm}(21027.77 \text { d.f }) ; \\
& \operatorname{mean} m_{1 ; \beta, e}^{D T}=4.4 \mathrm{~mm} ; \\
- & \min m_{1 ; \beta, n}^{D T}=4.6 \mathrm{~mm} \quad(12928.44 \mathrm{~d} . \mathrm{f}) ; \\
& \max m_{1 ; \beta, n}^{D T}=9.0 \mathrm{~mm}(20815.24 \mathrm{~d} . \mathrm{f}) ; \\
& \operatorname{mean} m_{1 ; \beta, n}^{D T}=6.0 \mathrm{~mm} ; \\
- & \min m_{1 ; \beta, u}^{D T}=9.1 \mathrm{~mm}(19106.55 \mathrm{~d} . \mathrm{f}) ; \\
& \max m_{1 ; \beta, u}^{D T}=20.3 \mathrm{~mm}(22763.77 \text { d.f }) ; \\
& \operatorname{mean} m_{1 ; \beta, u}^{D T}=12.5 \mathrm{~mm} .
\end{aligned}
$$

Nighttime period

$$
\begin{aligned}
&- \min m_{1 ; \beta, e}^{N T}=3.2 \mathrm{~mm}(13406.56 \mathrm{~d} . \mathrm{f}) ; \\
& \max m_{1 ; \beta, e}^{N T}=4.9 \mathrm{~mm}(16609.49 \mathrm{~d} . \mathrm{f}) ; \\
& \operatorname{mean} m_{1 ; \beta, e}^{N T}=4.0 \mathrm{~mm} ; \\
&-\min m_{1 ; \beta, n}^{N T}=4.7 \mathrm{~mm}(18597.80 \mathrm{~d} . \mathrm{f}) ; \\
& \max m_{1 ; \beta, n}^{N T}=7.3 \mathrm{~mm}(18783.11 \mathrm{~d} . \mathrm{f}) ; \\
& \operatorname{mean} m_{1 ; \beta, n}^{N T}=5.6 \mathrm{~mm} ; \\
&-\min m_{1 ; \beta, u}^{N T}=8.4 \mathrm{~mm}(16092.83 \mathrm{~d} . \mathrm{f}) ; \\
& \max m_{1 ; \beta, u}^{N T}=15.4 \mathrm{~mm}(19477.51 \mathrm{~d} . \mathrm{f}) ; \\
& \operatorname{mean} m_{1 ; \beta, u}^{N T}=11.3 \mathrm{~mm} .
\end{aligned}
$$

Joint effect of "near-field" multipath and receiver antenna phase center offset and variations:

Daytime period

$$
\begin{aligned}
&- \min m_{1 ; \alpha, e}^{D T}=1.5 \mathrm{~mm} \quad(507.88 \mathrm{~d} . \mathrm{f}) ; \\
& \max m_{1 ; \alpha, e}^{D T}=4.7 \mathrm{~mm}(728.60 \mathrm{~d} . \mathrm{f}) ; \\
& \operatorname{mean} m_{1 ; \alpha, e}^{D T}=2.9 \mathrm{~mm} ; \\
&-\min m_{1 ; \alpha, n}^{D T}=1.9 \mathrm{~mm}(701.42 \mathrm{~d} . \mathrm{f}) ; \\
& \max m_{1 ; \alpha, n}^{D T}=7.0 \mathrm{~mm}(856.69 \mathrm{~d} . \mathrm{f}) ; \\
& \operatorname{mean} m_{1 ; \alpha, n}^{D T}=4.5 \mathrm{~mm} ; \\
&-\min m_{1 ; \alpha, u}^{D T}=3.4 \mathrm{~mm}(739.53 \mathrm{~d} . \mathrm{f}) ; \\
& \max m_{1 ; \alpha, u}^{D T}=21.9 \mathrm{~mm}(759.82 \mathrm{~d} . \mathrm{f}) ; \\
& \operatorname{mean} m_{1 ; \alpha, u}^{D T}=13.0 \mathrm{~mm} .
\end{aligned}
$$

Nighttime period

$$
\begin{aligned}
& -\min m_{1 ; \alpha, e}^{N T}=0.8 \mathrm{~mm} \quad(691.85 \text { d.f }) ; \\
& \max m_{1 ; \alpha, e}^{N T}=3.8 \mathrm{~mm}(802.20 \text { d.f }) ; \\
& \operatorname{mean} m_{1 ; \alpha, e}^{N T}=2.5 \mathrm{~mm} ;
\end{aligned}
$$

Table 7. Hypothesis acceptance percentage by the relative coordinates $(e, n$ and $u$ ) for FF MP effect $(\beta)$, the joint effect of NF MP and RAPCOV $(\alpha 1)$ and the joint effect of TI $(\alpha 2)$

\begin{tabular}{|l|c|c|c|c|c|c|c|c|c|}
\hline & \multicolumn{3}{|c|}{$\beta$} & \multicolumn{2}{|c|}{$\alpha 1$} & \multicolumn{3}{c|}{$\alpha 2$} \\
\hline & $e$ & $n$ & $u$ & $e$ & $n$ & $u$ & $e$ & $n$ & $u$ \\
\hline $\mathrm{H}_{a 1 ; 1(2)}$ & $81.3 \%$ & $56.3 \%$ & $83.3 \%$ & $64.6 \%$ & $62.5 \%$ & $72.9 \%$ & $46.7 \%$ & $46.8 \%$ & $39.6 \%$ \\
\hline $\mathrm{H}_{0 ; 1(2)}$ & $4.2 \%$ & $4.2 \%$ & $8.3 \%$ & $22.9 \%$ & $14.6 \%$ & $16.7 \%$ & $11.1 \%$ & $19.1 \%$ & $12.5 \%$ \\
\hline $\mathrm{H}_{a 2 ; 1(2)}$ & $14.6 \%$ & $39.6 \%$ & $8.3 \%$ & $12.5 \%$ & $22.9 \%$ & $10.4 \%$ & $42.2 \%$ & $34.0 \%$ & $47.9 \%$ \\
\hline
\end{tabular}




$$
\begin{aligned}
- & \min m_{1 ; \alpha, n}^{N T}=2.1 \mathrm{~mm}(522.22 \text { d.f); } \\
& \max m_{1 ; \alpha, n}^{N T}=5.0 \mathrm{~mm}(732.56 \text { d.f); } \\
& \operatorname{mean} m_{1 ; \alpha, n}^{N T}=3.7 \mathrm{~mm} ; \\
- & \min m_{1 ; \alpha, u}^{N T}=3.1 \mathrm{~mm}(687.14 \text { d.f); } \\
& \max m_{1 ; \alpha, u}^{N T}=15.8 \mathrm{~mm} \text { (710.69 d.f); } \\
& \operatorname{mean} m_{1 ; \alpha, u}^{N T}=10.7 \mathrm{~mm} .
\end{aligned}
$$

Joint effect of tropospheric and ionospheric refraction:

Daytime period

$$
\begin{aligned}
&- \min m_{2 ; \alpha, e}^{D T}=0.0116 \mathrm{~mm} / \mathrm{km}(798.60 \mathrm{~d} . \mathrm{f}) ; \\
& \max m_{2 ; \alpha, e}^{D T}=0.3282 \mathrm{~mm} / \mathrm{km} \quad(859.90 \mathrm{~d} . \mathrm{f}) ; \\
& \operatorname{mean} m_{2 ; \alpha, e}^{D T}=0.1170 \mathrm{~mm} / \mathrm{km} ; \\
&-\min m_{2 ; \alpha, n}^{D T}=0.0103 \mathrm{~mm} / \mathrm{km}(866.85 \mathrm{~d} . \mathrm{f}) ; \\
& \max m_{2 ; \alpha, n}^{D T}=0.2365 \mathrm{~mm} / \mathrm{km}(856.69 \mathrm{~d} . \mathrm{f}) ; \\
& \operatorname{mean} m_{2 ; \alpha, n}^{D T}=0.1020 \mathrm{~mm} / \mathrm{km} ; \\
&-\min m_{2 ; \alpha, u}^{D T}=0.1222 \mathrm{~mm} / \mathrm{km} \quad(905.91 \mathrm{~d} . \mathrm{f}) ; \\
& \max m_{2 ; \alpha, u}^{D T}=0.7818 \mathrm{~mm} / \mathrm{km} \quad(944.97 \mathrm{~d} . \mathrm{f}) ; \\
& \operatorname{mean} m_{2 ; \alpha, u}^{D T}=0.3675 \mathrm{~mm} / \mathrm{km} .
\end{aligned}
$$

Nighttime period

$$
\begin{aligned}
&- \min m_{2 ; \alpha, e}^{N T}=0.0118 \mathrm{~mm} / \mathrm{km}(796.24 \mathrm{~d} . \mathrm{f}) ; \\
& \max m_{2 ; \alpha, e}^{N T}=0.2734 \mathrm{~mm} / \mathrm{km}(538.58 \mathrm{~d} . \mathrm{f}) ; \\
& \operatorname{mean} m_{2 ; \alpha, e}^{N T}=0.1149 \mathrm{~mm} / \mathrm{km} ; \\
&-\min m_{2 ; \alpha, n}^{N T}=0.0097 \mathrm{~mm} / \mathrm{km} \text { (792.19 d.f); } \\
& \max m_{2 ; \alpha, n}^{N T}=0.2289 \mathrm{~mm} / \mathrm{km}(846.56 \mathrm{~d} . \mathrm{f}) ; \\
& \operatorname{mean} m_{2 ; \alpha, n}^{N T}=0.0873 \mathrm{~mm} / \mathrm{km} ; \\
&-\min m_{2 ; \alpha, u}^{N T}=0.0752 \mathrm{~mm} / \mathrm{km}(633.06 \mathrm{~d} . \mathrm{f}) ; \\
& \max m_{2 ; \alpha, u}^{N T}=0.6315 \mathrm{~mm} / \mathrm{km}(853.08 \mathrm{~d} . \mathrm{f}) ; \\
& \operatorname{mean} m_{2 ; \alpha, u}^{N T}=0.3806 \mathrm{~mm} / \mathrm{km} .
\end{aligned}
$$

The results obtained in this study can be very useful in optimal planning of experiments, as well as in engineering works where relative GPS positioning is used.

\section{References}

Anđić, D. (2016). Variance components estimation of residual errors in GPS precise positioning. Geodetski vestnik, 60(3), 467-482.

https://doi.org/10.15292/geodetski-vestnik.2016.03.467-482

Anđić, D. (2019a). Seasonal pattern in time series of variances of GPS residual errors ANOVA estimates. Geodetski vestnik, 63(2), 260-271.

https://doi.org/10.15292/geodetski-vestnik.2019.02.260-271

Anđić, D. (2019b). Određivanje komponenti disperzija vremenski varijabilnih grešaka u GPS određivanju koordinata [Estimation of time-variable error variance components in GPS determination of coordinates] [Doctoral dissertation]. University of Belgrade, Serbia (in Serbian). https://doi.org/10.13140/RG.2.2.19498.36808/2

Deng, L., Jiang, W., Li, Z., Chen, H., Wang, K., \& Ma, Y. (2016). Assesment of second- and third-order ionospheric effects on regional networks: Case study in China with longer CMONOC GPS coordinate series. Journal of Geodesy, 91(2), 1-21. https://doi.org/10.1007/s00190-016-0957-y

Elsobeiey, M., \& El-Diasty, M. (2016). Impact of tropospheric delay gradients on total tropospheric delay and precise point positioning. International Journal of Geosciences, 7(5), 645654. https://doi.org/10.4236/ijg.2016.75050

Han, K., Tang, C., \& Deng, Z. (2019). A new method for multipath filtering in GPS static high-precision positioning. Sensors, 19(12), 2704. https://doi.org/10.3390/s19122704

Hu, Z., Zhao, Q., Chen, G., Wang, G., Dai, Z., \& Li, T. (2015). First results of field absolute calibration of the GPS receiver antenna at Wuhan University. Sensors, 15(11), 28717-28731. https://doi.org/10.3390/s151128717

Jadviščok, P., Ovesná, G., \& Konečný, M. (2016). Multipath and its manifestations in the real environment of geodetic practice. Geodesy and Cartography, 42(2), 47-52. https://doi.org/10.3846/20296991.2016.1198573

Juni, I., \& Rózsa, S. (2019). Validation of a new model for the estimation of residual tropospheric delay error under extreme weather conditions. Periodica Polytechnica Civil Engineering, 63(1), 121-129. https://doi.org/10.3311/PPci.12132

Kallio, U., Koivula, H., Lahtinen, S., Nikkonen, V., \& Poutanen, M. (2019). Validating and comparing GNSS antenna calibrations. Journal of Geodesy, 93, 1-18. https://doi.org/10.1007/s00190-018-1134-2

Klos, A., Hunegnaw, A., Teferle, F. N., Abraha, K. E., Ahmed, F., \& Bogusz, J. (2018). Statistical significance of trends in Zenith Wet Delay from re-processed GPS solutions. GPS Solutions, 22(2), 51. https://doi.org/10.1007/s10291-018-0717-y

Snedecor, G. W., \& Cochran, W. G. (1989). Statistical methods (8th ed.). Ames.

Zhou, Y., Kuang, C., \& Cai, C. (2018). Analysis of high-order ionospheric effects on GNSS precise point positioning in the China area. Survey Review, 51(368), 442-449. https://doi.org/10.1080/00396265.2018.1478483 